\title{
Dohle colgajo lihre para reconstrucción mandibular compleja: osteocutáneo de peroné y antebraquial radial. Caso clínico
}

\section{Double free flap for complex mandihular reconstruction: osteocutaneous fibula flap and radial forearm flap. Gase report}

\author{
Diego ROBALINO-TORRES*, Daysi CASTRO-CHÁVEZ ${ }^{* *}$, Fernando SANDOVAL-PORTILA ${ }^{* * *}$, \\ Carlos FREIRE-TORRES ${ }^{\star * * *}$, Armando SERRANO-GONZÁLEZ-RUBIO*****
}

Resumen

La reconstrucción mandibular con doble colgajo libre vascularizado es inusual en la reconstrucción de cabeza y cuello.

El presente artículo describe el caso de una paciente de 24 años de edad con osteosarcoma condroblástico de alto grado de piso de boca y hemimandíbula izquierda. Se realizó hemimandibulectomía amplia con resección de piel submandibular, procedimiento que dejó un defecto complejo que incluía hueso, mucosa y piel.

La resección tumoral y la reconstrucción del defecto fue llevada a cabo en un solo procedimiento quirúrgico a través de 2 colgajos libres: uno osteocutáneo de peroné y otro antebraquial radial.

Esta reconstrucción permitió alcanzar una función masticatoria adecuada, un contorno mandibular estético y una fonación normal.

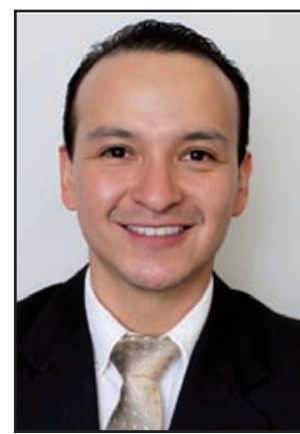

Robalino Torres, D.
Mandibular reconstruction with double vascularized free flap in the reconstruction of head and neck is unusual.

This article describes the case of a female patient 24 years old with a high-grade chondroblastic osteosarcoma of the floor of the mouth and of the left hemimandible. Hemimandibulectomy was performed with a wide resection of submandibular skin, leaving a complex defect which included bone, mucosa and skin.

Tumor resection and reconstruction of the defect was performed in a single surgical procedure through 2 free flaps: a free osteocutaneous fibula flap and radial forearm flap.

The reconstruction allowed to achieve an adequate chewing function, aesthetic mandibular contour and normal phonation.

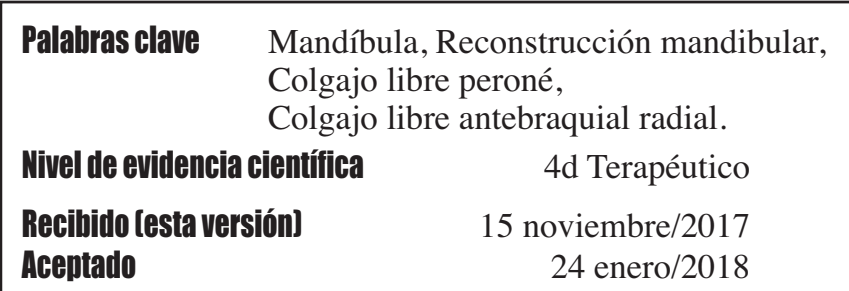

$\begin{array}{ll}\text { Key words } & \text { Mandible, Mandibular reconstruction, } \\ & \text { Fibula free flap, } \\ & \text { Radial foramen free flap. }\end{array}$

Level of evidence

Received [this version]

Accepted
$4 d$ Therapeutic

15 november/2017

24 january/2017

Conflicto de intereses: los autores declaran no tener ningún interés financiero relacionado con el contenido de este artículo.

* Residente de Postgrado de Cirugía Oral y Maxilofacial de la Universidad San Francisco de Quito, Ecuador.

** Residente asistencial del Hospital Oncológico Solón Espinoza Ayala, SOLCA, Núcleo de Quito, Ecuador.

*** Coordinador del Postgrado de Cirugía Oral y Maxilofacial de la Universidad San Francisco de Quito, Ecuador.

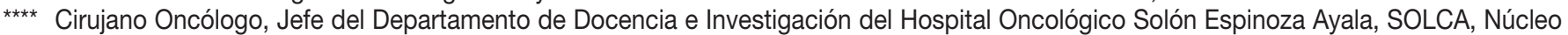
de Quito, Ecuador.

**** Cirujano Plástico, Jefe del Servicio de Cirugía Plástica y Reconstructiva del Hospital Oncológico Solón Espinoza Ayala, SOLCA, Núcleo de Quito, Ecuador. 


\section{Introducción}

El abordaje de las enfermedades oncológicas de la cabeza y el cuello ha sufrido cambios en las últimas dos décadas que incluyen avances en imagen, terapia adyuvante, técnica quirúrgica y una mejor comprensión de la anatomía. De esta forma se han desarrollado técnicas quirúrgicas reconstructivas que en el pasado pudieron ser imposibles. ${ }^{(1)}$

La reconstrucción microquirúrgica contribuye al restablecimiento de la forma, de la función masticatoria, de la deglución, la fonación y la estética, mejorando la calidad de vida del paciente. La mayoría de los defectos ablativos mandibulares complejos resultan de grandes resecciones oncológicas a nivel de orofaringe y cavidad oral, de manera que estos pacientes a menudo padecen defectos complejos y amplios que requieren reconstrucción. ${ }^{(2)}$

El colgajo osteocutáneo libre de peroné para reconstrucción mandibular fue introducido por Hidalgo en $1989 .^{(3-5)}$ Los beneficios de este colgajo incluyen la intervención simultánea de 2 equipos quirúrgicos, suficiente estructura ósea con entre $25-30 \mathrm{~cm}$ de hueso que puede ser contorneado para la reconstrucción mandibular total en defectos que lo requieran, permite la colocación de implantes osteointegrados dando estabilidad y alta posibilidad de oseointegración, provoca poca morbilidad del sitio donador, tiene una vascularización fiable procedente de los vasos peroneos con un pedículo de buena longitud y una isla de piel que puede ser utilizada para reconstrucción intraoral o extraoral de acuerdo a los requerimientos de cada paciente..$^{(3,6,7)}$ Sin embargo, los colgajos individuales pueden no ser suficientes para algunos defectos demasiado grandes o para garantizar el cierre de los tejidos, el tamaño de la isla de piel que aporta el colgajo es limitada, y por ende no provee suficiente tejido blando, por lo que se necesita en estos casos un segundo colgajo de piel. ${ }^{(7)}$

El colgajo antebraquial radial fue descrito por Yang y col. en 1981. Debido a su capacidad de transferencia confiable, su buena vascularización y su tejido moldeable, entre otros beneficios, este colgajo se ha utilizado en la reconstrucción de cabeza y cuello. ${ }^{(8)}$
Presentamos un caso clínico en el que realizamos la reconstrucción de un defecto ablativo oromandibular amplio con 2 colgajos libres vascularizados: un colgajo osteocutáneo libre de peroné para la reconstrucción mandibular y del piso de la boca, y un colgajo antebraquial radial para la reconstrucción del defecto cutáneo en el tercio inferior facial.

\section{Caso clínico}

Paciente de sexo femenino de 24 años de edad, sin antecedentes clínicos de interés, que presenta un cuadro de 8 meses de evolución que se inicia con parestesia labial inferior izquierda. A los 3 meses aparece una lesión tumoral de $1.5 \mathrm{~cm}$ de diámetro en región alveolar mandibular y piso de boca del mismo lado.

En el examen físico observamos asimetría facial del tercio inferior izquierdo con aumento de volumen en la región submandibular (Fig. 1). En el examen intra-oral apreciamos una lesión ubicada en el reborde alveolar mandibular y piso de boca del lado izquierdo, de consistencia indurada, coloración blanquecina y rojiza, con bordes irregulares, dolorosa, no móvil, con desplazamiento de piezas dentarias y expansión de corticales (Fig. 2).

La tomografía computarizada (TC) de macizo facial sin contraste muestra la presencia de una masa tumoral heterogénea con densidad central necrótica y periferia sólida, que infiltra los músculos geniohioideo, milohioideo y geniogloso, desplazando estructuras hacia el lado derecho. Además encontramos infiltración irregular en la cortical ósea de la rama mandibular izquierda. En la resonancia magnética (RM) evidenciamos la presencia de una lesión ocupativa hiperintensa redondeada, de bordes irregulares, con contenido heterogéneo, de aproximadamente $9 \times 8 \times 9 \mathrm{~cm}$, que desplaza las estructuras del piso de boca del lado izquierdo y de la vía aérea con efecto de masa (Fig. 3).

La biopsia incisional reportó osteosarcoma condroblástico de alto grado, tras lo cual decidimos como tratamiento llevar a cabo una hemimandibulectomía más
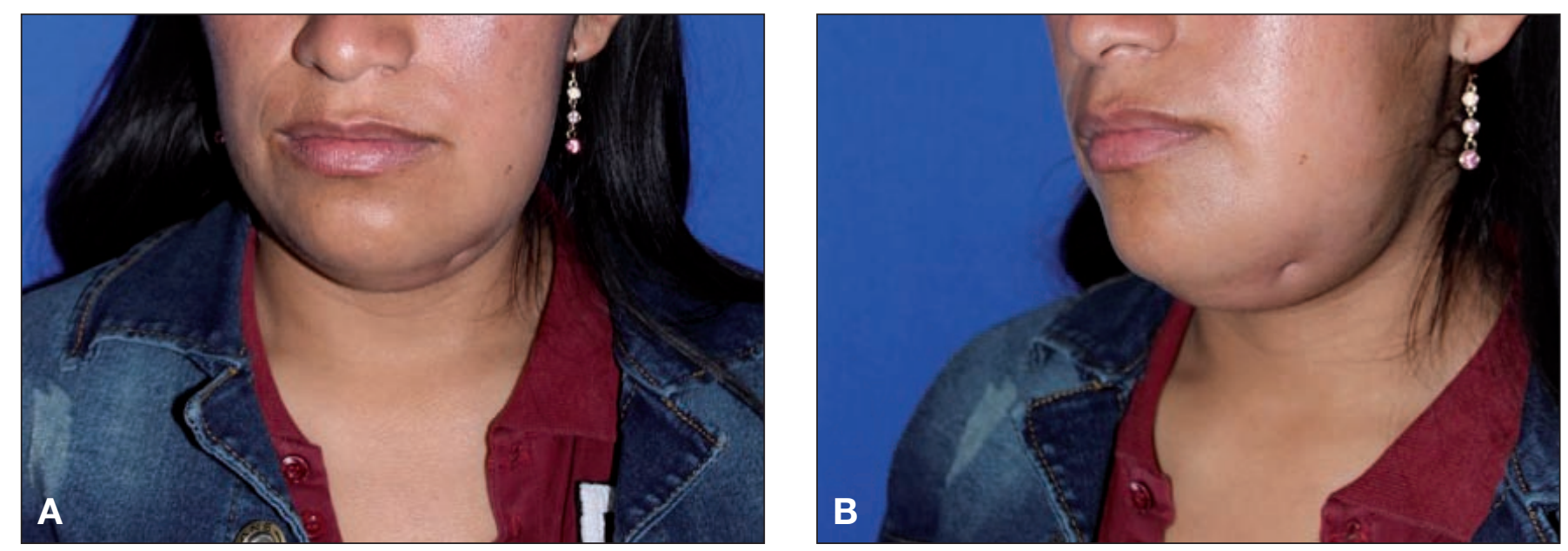

Fig. 1. Preoperatorio, mujer de 24 años con tumoración submandibular izquierda que causa asimetría. A. Vista frontal. B. Vista de medio perfil lado izquierdo. 


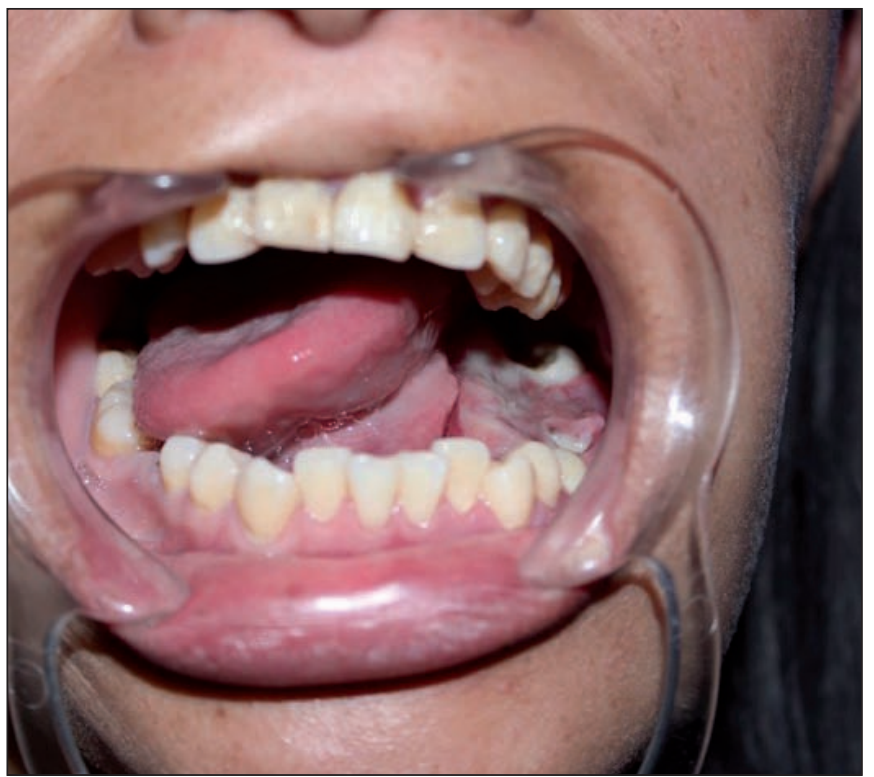

Fig. 2. Imagen intraoral preoperatoria de la lesión. Nótese lesión tumoral en reborde alveolar mandibular izquierdo, de coloración blanquecina y rojiza, con extensión al piso de la boca del mismo lado.

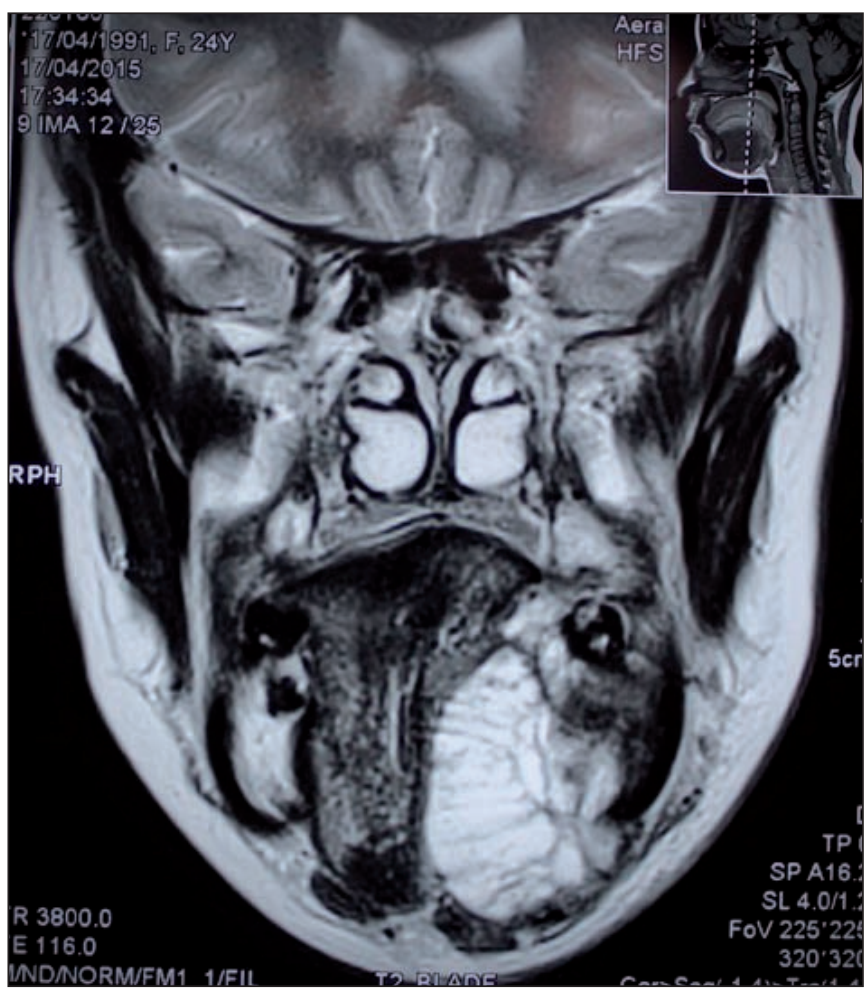

Fig. 3. Resonancia magnética. Corte coronal: lesión ocupativa hiperintensa, redondeada, de bordes irregulares, con contenido heterogéneo, que desplaza estructuras de piso de boca del lado izquierdo hasta línea media.

reconstrucción inmediata del defecto con un colgajo osteocutáneo libre de peroné para el segmento óseo mandibular y la mucosa del piso de boca, y reconstrucción del defecto de piel de la región facial con un colgajo antebraquial radial. Para la resección tumoral practicamos traqueostomía previa.

El procedimiento fue realizado por 2 equipos quirúrgicos que trabajaron simultáneamente. El primero estuvo formado por Cirugía Oncológica y el segundo por Cirugía Plástica y Cirugía Maxilofacial. El primer equipo realizó la traqueostomía, la resección oncológica y la preparación de los vasos receptores para los 2 colgajos libres: la arteria y vena faciales derecha e izquierda. El segundo equipo realizó la disección, el levantamiento del colgajo osteocutáneo libre de peroné, las osteotomías en el colgajo peroneo, su transporte, adaptación de tejidos blandos y duros para la reconstrucción mandibular, las anastomosis, la reconstrucción de la cubierta interna, el levantamiento del colgajo antebraquial radial, su trasporte, anastomosis y adaptación a la cubierta externa.

La resección oncológica consistió en una hemimandibulectomía del lado izquierdo, resección del piso de boca y disección cervical radical modificada tipo III del mismo lado, dejando un defecto amplio con dimensiones de $9.5 \mathrm{~cm}$ de longitud en sentido anteroposterior, $8 \mathrm{~cm}$ de altura y $6 \mathrm{~cm}$ de profundidad, de tejidos blandos y hueso (Fig. 4A).

La lesión fue enviada para estudio histopatológico (Fig. 4B).

Seguidamente, levantamos un colgajo osteocutáneo libre de peroné de $10 \mathrm{~cm}$ con una isla de piel de $13 \times 17 \mathrm{~cm}$ con su pedículo vascular. Realizamos 2 osteotomías en la parte proximal del peroné, una para la sínfisis y parasínfisis y otra para la reconstrucción del cuerpo mandibular.

Empleamos $3 \mathrm{~cm}$ de hueso para la reconstrucción de sínfisis y parasínfisis y $5 \mathrm{~cm}$ para la reconstrucción del cuerpo mandibular del lado izquierdo. Fijamos los segmentos óseos con 2 placas de osteosíntesis de 4 orificios de $2.0 \mathrm{~mm}$ con tornillos de $2.0 \times 7 \mathrm{~mm}$ de longitud; y 1 placa de 4 orificios de $2.4 \mathrm{~mm}$ con tornillos de $2.0 \mathrm{~mm} \mathrm{x}$ $10 \mathrm{~mm}$. Replegamos la isla de piel hacia dentro para formar el piso de boca y fijamos los segmentos de peroné a la mandíbula (Fig. 5).

Anastomosamos la arteria peronea de forma términoterminal a la arteria facial izquierda, y la vena peronea también de forma término-terminal al tronco tirolinguofaringofacial. Cubrimos el sitio donante con un injerto de piel tomado del muslo izquierdo.

Levantamos un colgajo libre antebraquial radial del brazo izquierdo tras marcar unas dimensiones de $6 \times 12 \mathrm{~cm}$, que empleamos para la reconstrucción del defecto de piel submandibular que abarcaba desde la línea media hasta el ángulo mandibular izquierdo. Hicimos la anastomosis de este colgajo término-terminalmente a la arteria y venas faciales del lado derecho. Llevamos a cabo el cierre de la zona donante del colgajo antebraquial radial mediante cobertura con injerto de piel de espesor parcial tomado de la zona anterior del muslo, suturado y fijado con un apósito de Brown.

Practicamos monitorización postoperatoria del colgajo cada 2 horas, comprobando la correcta perfusión y supervivencia de los colgajos. Tras la cirugía, la paciente permaneció en Unidad de Cuidados Intensivos (UCI) durante 72 horas, de las cuales se mantuvo bajo sedación profunda durante las primeras 24 horas para evitar movimientos bruscos del sitio de anastomosis; pasadas 72 

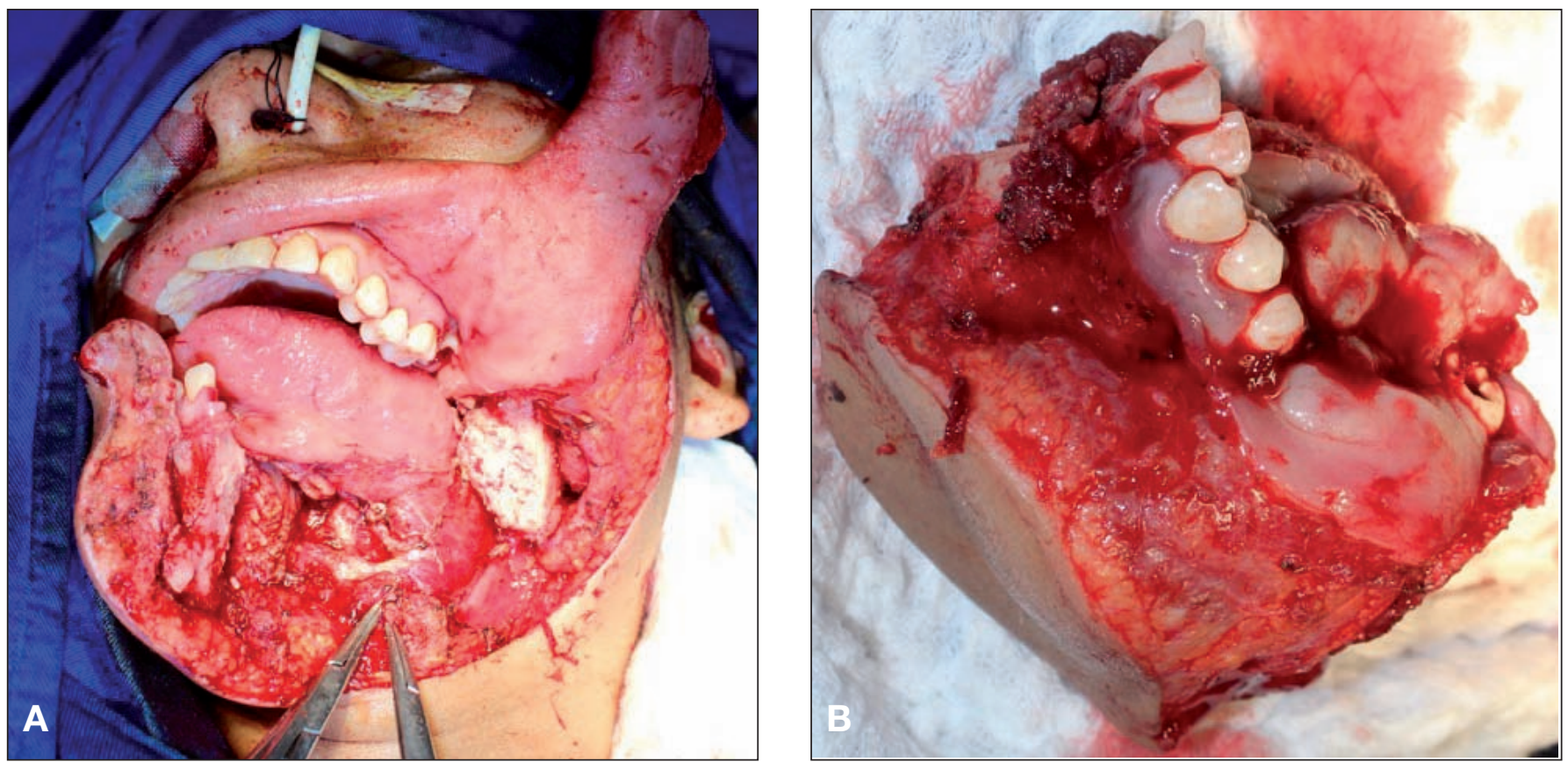

Fig. 4. Intraoperatoro. A. Defecto complejo de hueso, mucosa y tejidos blandos tras resección amplia mediante hemimandibulectomía izquierda B. Lesión tumoral de región mandibular izquierda para estudio histopatológico.

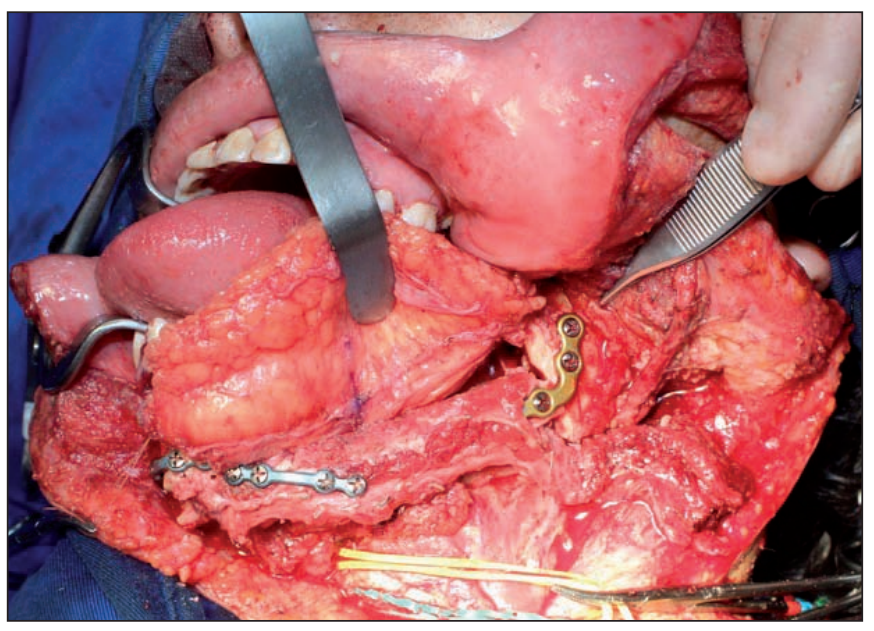

Fig. 5. Reconstrucción mandibular: segmentos óseos fijados al remanente mandibular con placas y tornillos de osteosíntesis. Isla de piel separada hacia arriba.

horas fue trasladada al área de hospitalización, donde permaneció durante 6 días siendo dada de alta al $9^{\circ}$ día de postoperatorio.

Empleamos como medicamento anticoagulante enoxaparina de $40 \mathrm{mg}$ en administración subcutánea, $1 \mathrm{vez}$ al día durante 14 días, y como antibiótico ampicilina + antibetalactámico de amplio espectro a dosis de $1.5 \mathrm{mg}$ en administración intravenosa cada 6 horas durante 7 días.

Durante la hospitalización, al $6^{\circ}$ día de postoperatorio la paciente comenzó a recibir líquidos claros por vía oral y dieta blanda al $7^{\circ}$ día mediante papillas y suplementos, presentando un buen estado nutricional. Inició deambulación a los 15 días de la cirugía.

Una vez dada de alta hospitalaria realizamos controles periódicos cada 8 días durante los 2 primeros meses y a partir del tercer mes, controles 1 vez por mes. La paciente fue remitida a tratamiento quimioterápico.
No llevamos a cabo ningún tipo de comprobación intraoperatoria. El resultado del estudio histopatológico postoperatorio reportó osteosarcoma condroblástico de alto grado en base a la pieza quirúrgica resecada de dimensiones 7 × 6 × $9.5 \mathrm{~cm}$, cubierta por elipse de piel marrón de 7 x $5.5 \mathrm{~cm}$., en cuyo borde anterior se observó un segmento óseo blanquecino rodeado por tejido blando sobre el que se identificaron 7 piezas dentales ( 3 incisivos, 1 canino, 2 premolares y un molar) en mal estado; en medio de estas se notó una lesión tumoral firme de 2.7 x 4 x $6 \mathrm{~cm}$, con expansión al piso de boca. El borde anterior distó $2.5 \mathrm{~cm}$ de la lesión tumoral. En el borde posterior de la pieza resecada se identificó un segmento irregular triangular de tejido óseo cubierto por tejido adiposo y muscular, además de 2 estructuras rosadas lobuladas de $3.5 \times 2 \times 1.4 \mathrm{~cm}$. y $1.5 \times 1 \times 0.6 \mathrm{~cm}$; el borde posterior distó $1.8 \mathrm{~cm}$ de la lesión tumoral. En los bordes laterales se identificó una lesión encapsulada de aspecto cartilaginoso blanco nacarado en el centro el tejido duro con $5.3 \mathrm{~cm}$. de diámetro, y los bordes interno y externo distaron $0.5 \mathrm{~cm}$ y $0.3 \mathrm{~cm}$ respectivamente de la lesión tumoral.

Realizamos una radiografía postoperatoria de control 30 días después del procedimiento quirúrgico, comprobando la reconstrucción mandibular (Fig. 6A). Tras 6 meses de evolución la fonación era normal, la paciente masticaba alimentos sólidos con normalidad por el lado no afectado y no hubo fístulas ni exposición de hueso o del material de osteosíntesis. La oclusión se desplazó aproximadamente $2 \mathrm{~mm}$ hacia el lado derecho, considerando la falta de musculatura del lado afectado (Fig. 6B).

El defecto quedó de esta forma cubierto en su totalidad y se devolvió el contorno mandibular (Fig. 7). Tampoco observamos complicaciones en los sitios donantes de ambos colgajos. 

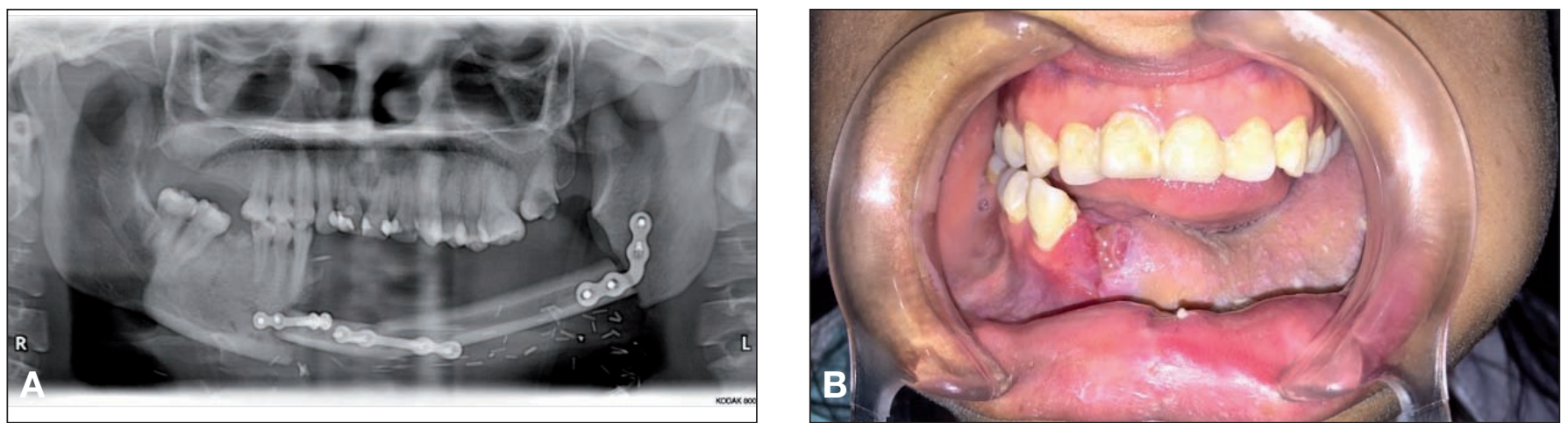

Fig. 6. A. Ortopantomografía de control a los 30 días de la cirugía. B. Fotografía postoperatoria intraoral a los 30 días del procedimiento: isla de piel del colgajo libre de peroné en el lugar del defecto. Oclusión con desplazamiento mandibular hacia la derecha.
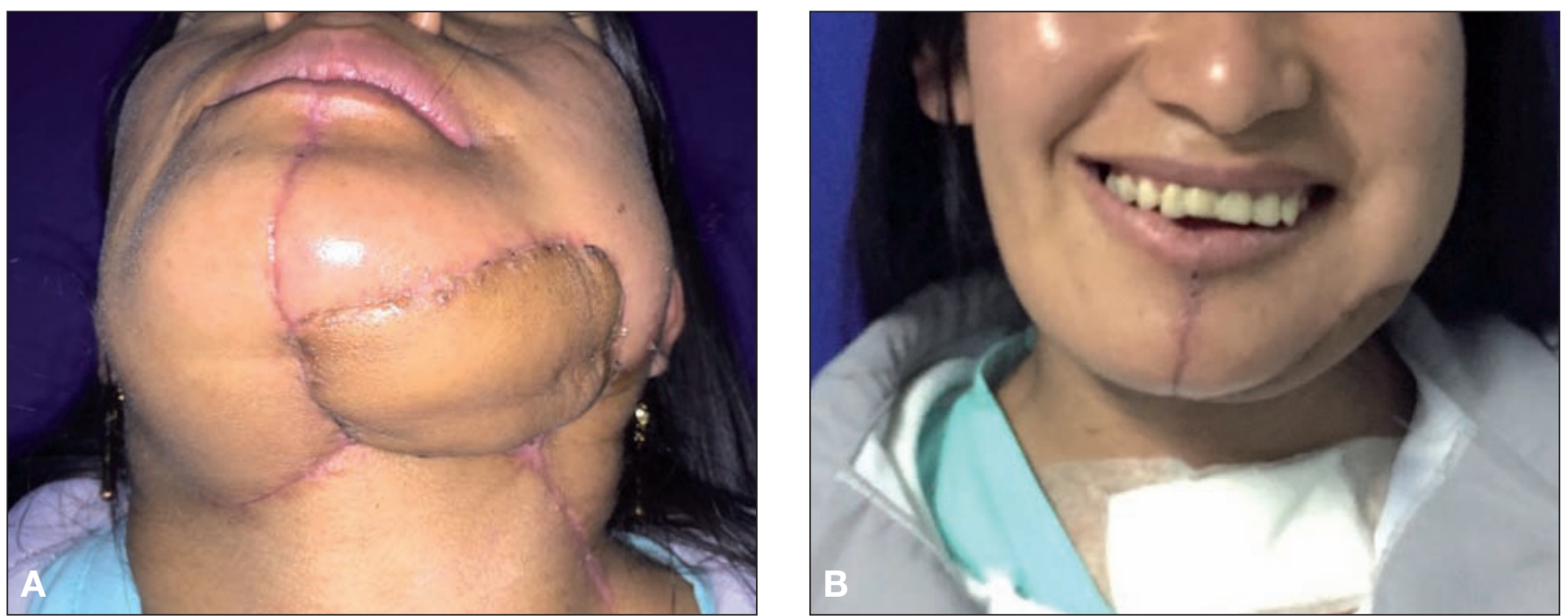

Fig. 7. Fotografías extraorales a los 30 días de la reconstrucción. A. Vista desde ángulo inferior. B. Vista frontal: se mantiene la continuidad del contorno mandibular.

En el control realizado a los 7 meses de postoperatorio, la paciente acudió refiriendo dolor en la región mandibular izquierda. El examen clínico intraoral evidenció la presencia de una lesión ulcerosa en la mucosa de la región retromolar izquierda, por lo cual se interconsultó al Servicio de Cabeza y Cuello y de Cirugía Maxilofacial de nuestro centro hospitalario, que indicó realizar una TC de control en la cual encontramos una masa osteolítica en la región posterior mandibular con cortical externa erosionada y ligera expansión a rama mandibular izquierda.

Llevamos a cabo una biopsia de dicha lesión con resultado de osteosarcoma condroblástico de alto grado. Al presentarse la recidiva, los nuevos estudios de imagen determinaron evolución desfavorable debido a la rápida invasión de la lesión hacia la base del cráneo con compromiso importante de estructuras anatómicas que impedían su resección. La paciente se mantuvo 9 meses tras detectar la recidiva con tratamiento paliativo y de terapia de dolor, y lamentablemente falleció a los 21 meses de la cirugía.

\section{Discusión}

En el pasado, los defectos faciales por resecciones oncológicas se consideraban un gran desafío debido a las limitadas opciones de reconstrucción con colgajos pediculados. Los avances actuales de la Microcirugía permiten la reconstrucción de defectos complejos y amplios, tanto de tejidos blandos como de hueso. ${ }^{(4.9)}$

De acuerdo a Laure y col. ${ }^{(9)}$ los 3 principales colgajos libres usados para la reconstrucción de defectos faciales con requerimiento de hueso son el colgajo de cresta iliaca, el de cresta escapular y el de peroné. El uso del colgajo de cresta iliaca presenta mayor morbilidad del sitio donante en comparación con el colgajo libre de peroné. El colgajo libre de cresta escapular solo facilita $12 \mathrm{~cm}$ de longitud y no permite el trabajo simultáneo de 2 equipos quirúrgicos, mientras que el colgajo libre de peroné está reconocido como el procedimiento más conveniente para la reconstrucción de defectos mandibulares amplios, permitiendo la intervención simultánea de 2 equipos. En nuestro caso, el levantamiento del colgajo libre de peroné para la reconstrucción del defecto óseo mandibular y del defecto amplio de piso de boca con la isla de piel, se realizó al mismo tiempo que la resección tumoral. El colgajo an- 
tebraquial radial fue levantado por el equipo de Cirugía Plástica de medial hacia lateral y de proximal hacia distal, mientras que el equipo de Cirugía Maxilofacial realizaba la adaptación del material de osteosíntesis y la reconstrucción mandibular. Escogimos este colgajo para la reconstrucción del defecto de piel que se produjo tras la resección tumoral en el lado izquierdo de la cara.

Mo y col. ${ }^{(7)}$ en su estudio de reconstrucción con colgajos dobles en 20 pacientes, afirman que las indicaciones para el uso de colgajos libres dobles para la reconstrucción son: la presencia de defectos extensos compuestos que afectan el hueso, a la mucosa oral, a la piel externa y al tejido blando, que no puede ser reconstruida adecuadamente con un colgajo individual; la falta de cobertura amplia en defectos intraorales donde un colgajo cutáneo o miocutáneo no es suficiente; y la dificultad de adaptación con un colgajo individual debido a la extensión y conformación tridimensional del defecto. En nuestro caso coincidimos en el uso de doble colgajo, que fue necesario para cubrir el defecto amplio de tejido blando submandibular resecado con el tumor y a la vez obtener el recubrimiento ideal para el tejido óseo utilizado en la reconstrucción de la mandíbula.

Es interesante destacar en nuestra presentación que la cirugía fue llevada a cabo por un grupo multidisciplinario formado por Cirugía Plástica y Reconstructiva, Cirugía Maxilofacial y Cirugía Oncológica. Antes de la cirugía se reunió un comité de tumores con las especialidades quirúrgicas mencionadas y las especialidades clínicas de Anestesiología, Oncología y Medicina Interna. Una vez determinado que la necesidad de resección quirúrgica incluía tejidos blandos de la cubierta facial submandibular izquierda y la resección de piso de boca y mandíbula, se decidió realizar una reconstrucción con doble colgajo libre, lo cual llevó a formar equipos que trabajaran de forma simultánea. Asi, mientras Cirugía Oncológica realizaba la resección de la lesión, Cirugía Plástica realizó el levantamiento del colgajo osteocutáneo de peroné; una vez que el colgajo estuvo levantado, Cirugía Maxilofacial realizó las osteotomías en el colgajo de peroné de acuerdo a las medidas del defecto creado en la mandíbula y Cirugía Plástica empezó el levantamiento del colgajo antebraquial radial. Terminada la resección de tumor, Cirugía Plástica en conjunto con Cirugía Maxilofacial trasportaron el colgajo osteocutáneo de peroné al cuello, realizaron la adaptación de los segmentos óseos con material de osteosíntesis, la adaptación de los tejidos blandos para recubrimiento interno y las anastomosis microquirúrgicas. Posteriormente, $\mathrm{Ci}$ rugía Plástica terminó el levantamiento del colgajo antebraquial radial y en conjunto con Cirugía Maxilofacial realizaron el recubrimiento externo y las anastomosis microquirúrgicas del segundo colgajo.

Laure y col..$^{(9)}$ mencionan que Ozkan y Sandove reportaron casos de reconstrucción simultánea de defectos maxilares y mandibulares con colgajos osteocutáneos li- bres de peroné combinados con colgajos anterolateral de muslo o antebraquial radial; no obstante, en un caso publicado por Laure y col., prefieren no usar un segundo colgajo libre para cubrir la piel o los defectos de la mucosa debido a los mayores riesgos de morbilidad usando dos colgajos, por lo cual prefieren emplear una isla de piel muy amplia en el colgajo libre de peroné.

Según Collin y col. ${ }^{(6)}$ existen problemas asociados a la disección de un pedículo de gran longitud en el colgajo libre de peroné, que incluyen obstrucción de los vasos peroneos en la parte proximal del peroné, riesgo de daño a estructuras durante la disección e inapropiada posición de las osteotomías que conducen a la longitud de la reducción del pedículo. En nuestro caso clínico no hubo problemas en la disección y obtuvimos un pedículo de $15 \mathrm{~cm}$ de longitud que nos permitió realizar osteotomías, adaptación y anastomosis del colgajo osteocutáneo sin complicaciones, comprobando la viabilidad vascular del colgajo como normal dentro de las primeras 48 horas de la cirugía, con controles cada 2 horas mediante ecodoppler.

Papadopolus y col.$^{(10)}$ en su estudio de 10 años de experiencia en reconstrucción mandibular con una serie de 23 pacientes, observaron un aporte vascular seguro con un $100 \%$ de supervivencia de todos los colgajos; sin embargo presentaron complicaciones menores en el postoperatorio inmediato como necrosis de bordes de la isla de piel y revisión de anastomosis. A su vez la restauración de la oclusión postoperatoria permitió un masticación satisfactoria, un habla inteligible y tolerancia de dieta normal. En nuestro caso la oclusión se vio alterada, observando una mordida cruzada en el lado derecho cuya causa creemos que fue la resección amplia que incluyó gran parte de la musculatura encargada de mantener la mandíbula en relación con el maxilar y la ausencia de piezas dentarias. La posición del cóndilo dentro de la cavidad glenoidea se mantuvo funcional permitiendo movimiento, masticación, tolerancia a dieta normal y fonación normal. Probablemente, mantener la fijación máxilo-mandibular la mayor parte de tiempo posible durante el transoperatorio permitiría evitar un mayor grado de desplazamiento mandibular.

De acuerdo a Preidl y col., ${ }^{(1)}$ el porcentaje de éxito del colgajo antebraquial radial es del $95 \%$ con resultados de adecuada función. En nuestro caso usamos este colgajo para reconstruir el defecto de piel submandibular y cubrir el colgajo libre de peroné con éxito.

En nuestra experiencia con el colgajo antebraquial radial, que es uno de los más utilizados, lo hemos empleado para la reconstrucción de defectos faciales como los causados por maxilectomías subtotales y en defectos por resecciones amplias en tejidos blandos; no hemos tenido complicaciones mayores que impliquen la pérdida del colgajo.

En cuanto a la evolución del caso presentado, sabemos que el osteosarcoma es un tumor óseo primario de alto grado de malignidad, con una alta tasa de metástasis 
y recidiva local. ${ }^{(12)}$ Nuestra paciente fue diagnosticada de osteosarcoma condroblástico mandibular de alto grado, por lo cual optamos por realizar la resección radical de la lesión y posteriormente recibió quimioterapia coadyuvante; sin embargo, al $7^{\circ}$ mes de la reconstrucción evidenciamos la rápida recidiva del tumor hacia base de cráneo. De acuerdo a Weinfeld y Dudley, ${ }^{(13)}$ en su estudio sobre 94 casos, la supervivencia a los 5 años de los pacientes con osteosarcoma es generalmente pobre, correspondiendo en osteosarcomas mandibulares a un $25 \%$. Desgraciadamente, nuestra paciente no respondió al tratamiento oncológico, por lo que pasó a cuidados paliativos y a los 22 meses de su diagnóstico falleció en su hogar, pasando a ser parte de la baja tasa de supervivencia en pacientes con osteosarcoma mandibular de alto grado.

\section{Conclusiones}

En nuestro caso de reconstrucción concluimos que es de vital importancia realizar una planificación adecuada con el apoyo de un equipo multidisciplinario y tener una perspectiva tridimensional en el abordaje de las lesiones oncológicas mandibulares para obtener un resultado favorable funcional y estético en relación a todos los tejidos afectados, de modo que la reconstrucción permita mejorar la calidad de vida del paciente.

Las reconstrucciones microquirúrgicas en este tipo de defectos no son frecuentes en un país como el nuestro, que se encuentra en vías de desarrollo, por lo cual destacamos la importancia de la preparación y planificación mediante esta viable alternativa de reconstrucción.

\section{Dirección del autor}

Dr. Diego Alejandro Robalino Torres

SOLCA Núcleo de Quito

Hospital Oncológico Solón Espinoza Ayala

Av. Eloy Alfaro 5394 y Los Pinos

Quito, Ecuador

Correo electrónico: alejodr87@hotmail.com

\section{Bibliografía}

1. Neligan PC. Head and Neck Reconstruction. Plast Reconstr Surg. 2013;131(2):260e-269e.

2. Schultz BD, Sosin M, Nam A, Mohan R, Zhang P, Khalifian S, et al. Classification of Mandible Defects and Algorithm for Microvascular Reconstruction. Plast Reconstr Surg. 2015; 135(4):743e-754e.

3. Peng X, Mao C, Yu G, Guo C, Huang M, Zhang Y. Maxillary reconstruction with the free fibula flap. Plast Reconstr Surg. 2005;115(6):1562-1569.

4. Steel BJ, Cope MR. A Brief History of Vascularized Free Flaps in the Oral and Maxillofacial Region. J Oral Maxillofac Sur.; 2015;73(4):786-786.

5. Leiggener C, Messo E, Thor a., Zeilhofer HF, Hirsch JM. A selective laser sintering guide for transferring a virtual plan to real time surgery in composite mandibular reconstruction with free fibula osseous flaps. Int J Oral Maxillofac Surg. 2009; 38(2):187-192.

6. Collin T, Sugden P, Ahmed O, Ragbir M. Technical considerations of fibular osteocutaneous flap dissection. J Plast Reconstr Aesthetic Surg; 2008;61(12):1503-1506.

7. Mo KW, Vlantis A, Wong EW, Chiu T. Double free flaps for reconstruction of complex/composite defects in head and neck surgery. Hong Kong Med J. 2014;20(4):4-6.

8. Futran ND, Gal TJ, Farwell DG. Radial forearm free flap. Oral Maxillofac Surg Clin North Am. 2003;15(4):577-591.

9. Laure B, Sury F, Martin T, Chabut A, Goga D. Reconstruction of bony mandibular and maxillary defects with one single transfer of a free fibula osteocutaneous flap. J Plast Reconstr Aesthetic Surg. 2008;61(2):200-203.

10. Papadopulos NA, Schaff J, Sader R, Kovacs L, Deppe H, Kolk A, et al. Mandibular reconstruction with free osteofasciocutaneous fibula flap: A 10 years experience. Int. J. Care Injured 2008; 39(3):75-82.

11. Preidl RHM, Wehrhan F, Schlittenbauer T, Neukam FW, Stockmann P. Perioperative factors that influence the outcome of microsurgical reconstructions in craniomaxillofacial surgery. Br J Oral Maxillofac Surg. 2015;1-7.

12. Klein MJ, Siegal GP. Osteosarcoma Anatomic and Histologic Variants. Am J Clin Pathol. 2006;125:555-581.

13. Weinfield M, Dudley R. Osteogenic Sarcoma: A Follow-up Study of the Ninety-Four Cases Observed at the Massachusetts General Hospital from 1920 to 1960. J Bone Jt Surg. 1962;44 A(2):269-276. 